\title{
Characteristics of pediatric rhabdomyolysis and the associated risk factors for acute kidney injury: a retrospective multicenter study in Korea
}

\author{
Sukdong Yoo ${ }^{1}$, Min Hyun $\mathrm{Cho}^{2}$, Hee Sun Baek ${ }^{2}$, Ji Yeon Song ${ }^{1}$, Hye Sun Lee ${ }^{3}$, Eun Mi Yang ${ }^{4}$, Kee Hwan Yoo, \\ Su Jin Kim ${ }^{6}$, Jae II Shin ${ }^{7}$, Keum Hwa Lee ${ }^{7}$, Tae-Sun Ha ${ }^{8}$, Kyung Mi Jang ${ }^{9}$, Jung Won Lee ${ }^{10}$, Kee Hyuck Kim ${ }^{11}$, \\ Heeyeon $\mathrm{Cho}^{12}$, Mee Jeong Lee ${ }^{13}$, Jin-Soon Suh ${ }^{14}$, Kyoung Hee Han ${ }^{15}$, Hye Sun Hyun ${ }^{16}$, II-Soo Ha ${ }^{17}$, \\ Hae Il Cheong ${ }^{18}$, Hee Gyung Kang ${ }^{17}$, Mee Kyung Namgoong ${ }^{19}$, Hye-Kyung $\mathrm{Cho}^{20}$, Jae-Hyuk $\mathrm{Oh}^{21}$, \\ Sang Taek Lee ${ }^{22}$, Kyo Sun Kim ${ }^{23}$, Joo Hoon Lee ${ }^{24}$, Young Seo Park ${ }^{24}$, Seong Heon Kim ${ }^{1}$ \\ For further information on the authors' affiliations, see Additional information.
}

\begin{abstract}
Background: The clinical features of pediatric rhabdomyolysis differ from those of the adults with rhabdomyolysis; however, multicenter studies are lacking. This study aimed to investigate the characteristics of pediatric rhabdomyolysis and reveal the risk factors for acute kidney injury (AKI) in such cases.

Methods: This retrospective study analyzed the medical records of children and adolescents diagnosed with rhabdomyolysis at 23 hospitals in South Korea between January 2007 and December 2016.

Results: Among 880 patients, those aged 3 to 5 years old composed the largest subgroup (19.4\%), and all age subgroups were predominantly male. The incidence of AKI was $11.3 \%$. Neurological disorders (53.6\%) and infection (39.0\%) were the most common underlying disorder and cause of rhabdomyolysis, respectively. The median age at diagnosis in the AKI subgroup was older than that in the non-AKI subgroup (12.2 years vs. 8.0 years). There were no significant differences in body mass index, myalgia, dark-colored urine, or the number of causal factors between the two AKI-status subgroups. The multivariate logistic regression model indicated that the following factors were independently associated with AKI: multiorgan failure, presence of an underlying disorder, strong positive urine occult blood, increased aspartate aminotransferase and uric acid levels, and reduced calcium levels.

Conclusion: Our study revealed characteristic clinical and laboratory features of rhabdomyolysis in a Korean pediatric population and highlighted the risk factors for AKI in these cases. Our findings will contribute to a greater understanding of pediatric rhabdomyolysis and may enable early intervention against rhabdomyolysis-induced AKI.
\end{abstract}

Keywords: Creatine kinase, Etiology, Muscles, Renal insufficiency

Received: March 3, 2021; Revised: June 13, 2021; Accepted: June 20, 2021

Editor: Soo Wan Kim, Chonnam National University, Gwangju, Republic of Korea

Correspondence: Seong Heon Kim

Department of Pediatrics, Pusan National University School of Medicine, 20 Geumo-ro, Mulgeum-eup, Yangsan 50612, Republic of Korea.

E-mail: pedksh@gmail.com

ORCID: https://orcid.org/0000-0001-8003-3010

Copyright (C) 2021 by The Korean Society of Nephrology

(a) This is an Open Access article distributed under the terms of the Creative Commons Attribution Non-Commercial and No Derivatives License (http:// creativecommons.org/licenses/by-nc-nd/4.0/) which permits unrestricted non-commercial use, distribution of the material without any modifications, and reproduction in any medium, provided the original works properly cited. 


\section{Introduction}

Rhabdomyolysis is a syndrome involving skeletal muscle damage caused by conditions such as severe trauma, physical exertion, seizure, drug use, toxins, biological agents, metabolic disorders, prolonged immobilization, and genetic defects. Subsequently, the breakdown of the damaged skeletal muscle leads to the release of its contents into plasma, thereby causing complications, such as acute kidney injury (AKI), electrolyte disturbance, compartment syndrome, and disseminated intravascular coagulation $[1,2]$. AKI is the leading cause of death in patients with rhabdomyolysis. Myoglobin-induced renal toxicity leads to rhabdomyolysis-associated AKI because of increased oxidative stress, inflammation, endothelial dysfunction, vasoconstriction, tubular obstruction, and apoptosis [1,3].

Rhabdomyolysis appears more frequently among male individuals, African-Americans, those aged younger than 10 or older than 60 years, and those with body mass index (BMI) values of greater than $40 \mathrm{~kg} / \mathrm{m}^{2}$ [4]. However, the etiology of rhabdomyolysis and the incidence of AKI may vary depending on age [4-7]. Furthermore, large-scale multicenter studies on rhabdomyolysis and the associated risk factors for AKI in the pediatric population are scarce. The primary aim of this study was to investigate the characteristics of rhabdomyolysis in a Korean pediatric population. The secondary aim was to identify the risk factors for AKI among patients with rhabdomyolysis.

\section{Methods}

\section{Study design}

This retrospective study analyzed the medical records of children and adolescents diagnosed with and treated for rhabdomyolysis at 23 hospitals in South Korea from January 2007 to December 2016. This study was approved by the Institutional Review Board of Pusan National University School of Medicine and informed consent was waived due to the nature of the retrospective study (No. 05-2018-021).

\section{Study population}

Rhabdomyolysis was defined by a creatine kinase (CK) level of at least 1,000 U/L or myoglobin level of at least $100 \mathrm{ng} /$
mL. Patients eligible for inclusion were those aged between one month and 18 years who met the definition of having rhabdomyolysis $(n=1,046)$. Individuals were excluded from this study if they had myocarditis or myocardial infarction, had received cardiopulmonary resuscitation $(\mathrm{n}=118)$ or had insufficient medical records $(n=48)$. Patients with muscular dystrophy were included only if they had not yet been diagnosed with such $(n=18)$. All subjects $(n=880)$ were divided into two AKI-status subgroups (with or without AKI) for comparison (Fig. 1).

\section{Data collection}

Medical records were reviewed to observe the following: age at diagnosis, sex, anthropometric data, symptoms, underlying illnesses, causal factors and complications of rhabdomyolysis, laboratory results, methods of treatment, and outcomes. All individuals were divided into five age subgroups because the incidence of rhabdomyolysis and the rate of AKI are not linearly associated with age [6]. Demographic data such as height, weight, and BMI were transformed into z-scores using the 2017 Korean National Growth Charts for Children and Adolescents (https://knhanes.cdc.go.kr/knhanes/sub08/sub08_02.do) and lambda-mu-sigma method [8]. AKI was defined as an increase in serum creatinine of at least $0.3 \mathrm{mg} / \mathrm{dL}$ within 48 hours or at least 1.5 times the minimum creatinine value during the hospitalized stay, regardless of the time period [9], which was established according to the creatinine criteria of the Kidney Disease:

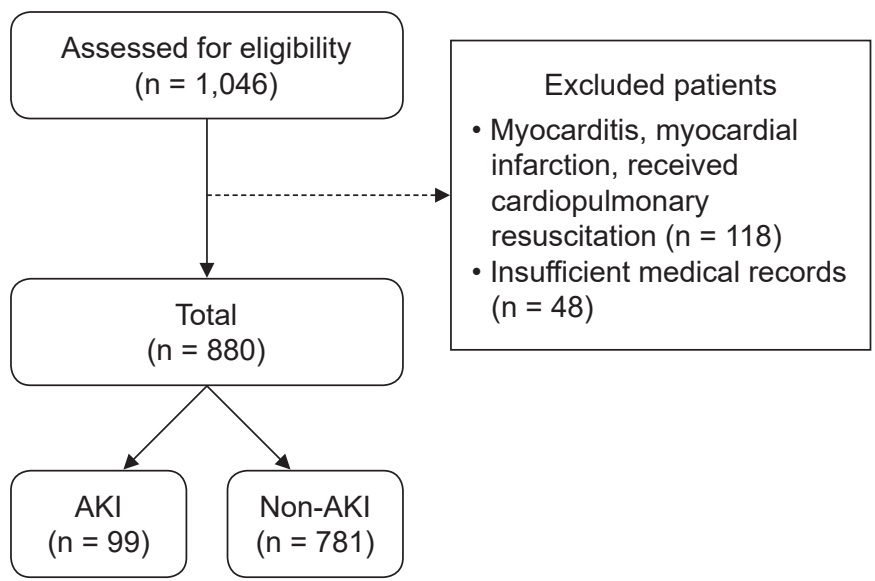

Figure 1. CONSORT flow diagram.

AKI, acute kidney injury. 
Improving Global Outcomes (KDIGO) guidelines [10]. Oliguria was defined as a urine output of less than $0.5 \mathrm{~mL} /$ $\mathrm{kg} / \mathrm{hr}$ for 12 hours. Urine output was determined based on details from the medical history, such as the time of last urination and medical records of urine output in the hospital. Multiorgan failure was defined as the failure of at least two of the major organ systems. Short-term and long-term outcomes were stratified based on a cutoff of 90 days after the date of AKI occurrence. The recovery duration of CK and myoglobin levels was defined as the period from the onset of rhabdomyolysis to the date of restoring the value in question to within the normal range. Volume overload was defined as the presence of pulmonary edema or peripheral edema. Chronic kidney disease was defined as a decrease in the glomerular filtration rate to less than $90 \mathrm{~mL} / \mathrm{min} / 1.73$ $\mathrm{m}^{2}$, which was calculated using the updated Schwartz formula as follows: $0.413 \times$ [height $(\mathrm{cm}) /$ serum creatinine $(\mathrm{mg} /$ $\mathrm{dL})$ ]. Laboratory parameters were analyzed by the following or equivalent methods: serum CK was analyzed by a CK $\mathrm{N}$-acetyl-cystein-activated procedure; serum myoglobin was analyzed by chemiluminescence immunoassay; qualitative and quantitative urine myoglobin levels were analyzed by ammonia sulfate precipitation testing with urine test strips and electrochemiluminescence immunoassay, respectively; and serum creatinine was analyzed using the modified Jaffe's kinetic method.

\section{Statistical analysis}

For the comparison of AKI and non-AKI subgroups, the Student $\mathrm{t}$ test or Mann-Whitney U test was performed for continuous variables, and the chi-squared test or Fisher exact test was performed for categorical variables. Multiple responses were allowed when surveying underlying diseases, causes, and complications of rhabdomyolysis. Statistical significance was set at $\mathrm{p}<0.05$.

We investigated whether AKI can be predicted by risk factors and initially accessible clinical information of patients with rhabdomyolysis. Multiple logistic regression analysis was performed to determine the independent risk factors associated with AKI. Candidate variables with p-values of less than 0.1 in the univariate analysis were entered into the model through variable selection by a stepwise method. Variance inflation factors were checked to eliminate multiple coherences. The results of the generated model were confirmed by the receiver-operating characteristic (ROC) curve and area under the ROC curve. All analyses were performed using R version 4.0.0 (R Foundation for Statistical Computing, Vienna, Austria).

\section{Results}

\section{Demographic data and clinical symptoms}

According to the study inclusion and exclusion criteria, 880 patients were included. The overall age distribution of these patients showed a bimodal peak (3-5 and 15-18 years, respectively). Among the five age subgroups, the largest one was those aged three to 5 years old in the total group and non-AKI subgroup, whereas it was 15 to 18 years old in the AKI subgroup. The median age at diagnosis was older in the AKI subgroup than in the non-AKI subgroup (12.2 years vs. 8.0 years; $\mathrm{p}<0.001$ ). There were 2.5 times more boys (ratio of boys:girls, 634:246), and all age subgroups were predominantly male (range of sex proportion, 2.0-3.6). Boys totaled $68.0 \%$ to $69.6 \%$ of patients younger than 12 years and $76.8 \%$ to $78.0 \%$ of those older than 12 years (Table 1 ).

Height, weight, and BMI z-scores did not significantly deviate from zero in the total rhabdomyolysis group. For those in the AKI subgroup, the actual height, weight, and BMI were higher, but their z-scores were not significantly different between the two subgroups. Body surface area was also higher in the AKI subgroup, but the z-scores could not be compared between the AKI-status subgroups because of a lack of data. Among the 880 patients, myalgia or muscle weakness was observed in $38.9 \%$ and dark-colored urine was present for $14.0 \%$. Although oliguria occurred in only $4.1 \%$ of all rhabdomyolysis cases, it affected one-third of the AKI subgroup. Only $3.4 \%$ of all rhabdomyolysis cases had two or more causes of rhabdomyolysis. There were no significant differences in myalgia or muscle weakness, dark-colored urine, or the number of causal factors between the two AKI-status subgroups (Table 1).

\section{Underlying disease}

Of the 880 patients, $28.6 \%$ had underlying diseases. A detailed list of underlying disorders is provided in the supplementary data (Supplementary Table 1, available online). Neurological disorders were the most common underlying 
Table 1. Characteristics of patients with acute rhabdomyolysis with or without AKI

\begin{tabular}{|c|c|c|c|c|}
\hline Variable & AKI $(n=99)$ & Non-AKI $(n=781)$ & Total $(n=880)$ & p-value \\
\hline Male sex & $65(65.7)$ & $569(72.9)$ & $634(72.0)$ & 0.17 \\
\hline Age at diagnosis (yr) & $12.2(6.0-15.1)$ & $8.0(4.0-13.4)$ & $8.0(4.0-14.0)$ & $<0.001$ \\
\hline $0-2$ & $12(12.1)$ & $140(17.9)$ & $152(17.3)$ & \\
\hline $3-5$ & $11(11.1)$ & $160(20.5)$ & $171(19.4)$ & \\
\hline $6-8$ & $9(9.1)$ & $119(15.2)$ & $128(14.5)$ & \\
\hline $9-11$ & $11(11.1)$ & $86(11.0)$ & $97(11.0)$ & \\
\hline $12-14$ & $22(22.2)$ & $142(18.2)$ & $164(18.6)$ & \\
\hline $15-18$ & $34(34.3)$ & $134(17.2)$ & $168(19.1)$ & \\
\hline Height z-score & 0.17 (-1.12 to 1.16$)$ & $0.29(-0.70$ to 1.35$)$ & $0.29(-0.76$ to 1.30$)$ & 0.57 \\
\hline Weight z-score & $0.01(-1.36$ to 0.84$)$ & $-0.12(-1.05$ to 0.78$)$ & $-0.11(-1.09$ to 0.78$)$ & 0.95 \\
\hline BMI z-score $(n=780)$ & $-0.03(-1.29$ to 0.86$)$ & -0.26 (-1.09 to 0.71) & $-0.23(-1.10$ to 0.72$)$ & 0.54 \\
\hline $\mathrm{BSA}^{\mathrm{b}}$ & $1.28(0.83-1.60)$ & $0.96(0.70-1.39)$ & $0.97(0.72-1.44)$ & 0.00 \\
\hline Myalgia or muscle weakness & $41(41.4)$ & $301(38.5)$ & $342(38.9)$ & 0.66 \\
\hline Dark-colored-urine & $20(20.2)$ & $103(13.2)$ & $123(14.0)$ & 0.08 \\
\hline Oliguria, yes & $36(36.4)$ & $0(0)$ & $36(4.1)$ & $<0.001$ \\
\hline No. of causal factors ${ }^{c}$ & & & & 0.07 \\
\hline Single & 92 (92.9) & $758(97.1)$ & $850(96.6)$ & \\
\hline Multiple & $7(7.1)$ & $23(2.9)$ & $30(3.4)$ & \\
\hline
\end{tabular}

Data are expressed as number (\%) or median (interquartile range).

AKI, acute kidney injury; BMI, body mass index; BSA, body surface area.

${ }^{a}$ National database of BMI only includes individuals older than 2 years. ${ }^{b}$ BSA z-score was not available in our study. ${ }^{c}$ Multiple responses allowed the collection of causal factor data.

disorder (53.6\%), followed by nephrological disorders, endocrinological and metabolic disorders, genetic and syndrome disorders, and cardiological disorders (Fig. 2A). The frequency of neurological disorders had a bimodal distribution and peaked in patients aged zero to 2 years and those aged 15 to 18 years, respectively. Most cardiological disorders were observed in patients younger than 9 years of age, whereas most psychiatric disorders were observed in those older than 9 years of age. No characteristic age distribution was identified for the other disorders (Fig. 2B). The underlying disorder that showed a significantly higher proportion in the AKI group included neurological disorders (26.3\% vs. $14.0 \% ; \mathrm{p}=0.002)$ and oncologic disorders $(4.0 \%$ vs. $0.6 \% ; \mathrm{p}$ $=0.008$ ). The proportion of total underlying disorders was higher in the AKI subgroup (53.5\% vs. $25.5 \%$; p < 0001).

\section{Etiology}

The most common cause of rhabdomyolysis was infection $(39.0 \%)$, followed by trauma or surgery $(21.1 \%)$, prolonged convulsions $(21.0 \%)$, and unknown causes (9.6\%). Other causes were diverse and heterogeneous, with a proportion of less than $3.0 \%$ (Fig. 3A). Influenza virus was the most common infectious cause. A detailed list of etiologies is shown in the supplementary data (Supplementary Table 2, available online).

Some causes of rhabdomyolysis were characterized by age; the greatest frequency of seizures and infections occurred at 0 to 2 years of age and 3 to 5 years of age, respectively. The frequency of trauma increased with age, with most cases occurring in patients aged 15 to 18 years (Fig. 3B-I). In the AKI group, although trauma was still prominent in older patients, the age pattern of infection and prolonged seizures in the total group became indistinctive. However, AKI was more frequently caused by relatively rare causes, such as drugs, multiorgan failure, cardiac arrhythmias, and metabolic disorders (Fig. 3B-II, III). Similarly, the differences in the frequency of infection (ratio of AKI:nonAKI subgroups, $36.1 \%: 40.8 \% ; \mathrm{p}=0.12$ ) and prolonged seizure (18.1\%:22.5\%; $\mathrm{p}=0.18)$ were not prominent, but those for multiorgan failure (12.4\%:1.2\%; $\mathrm{p}<0.001)$ and drugs (6.7\%:1.2\%; $\mathrm{p}<0.004)$ were significantly higher in the AKI subgroup. 

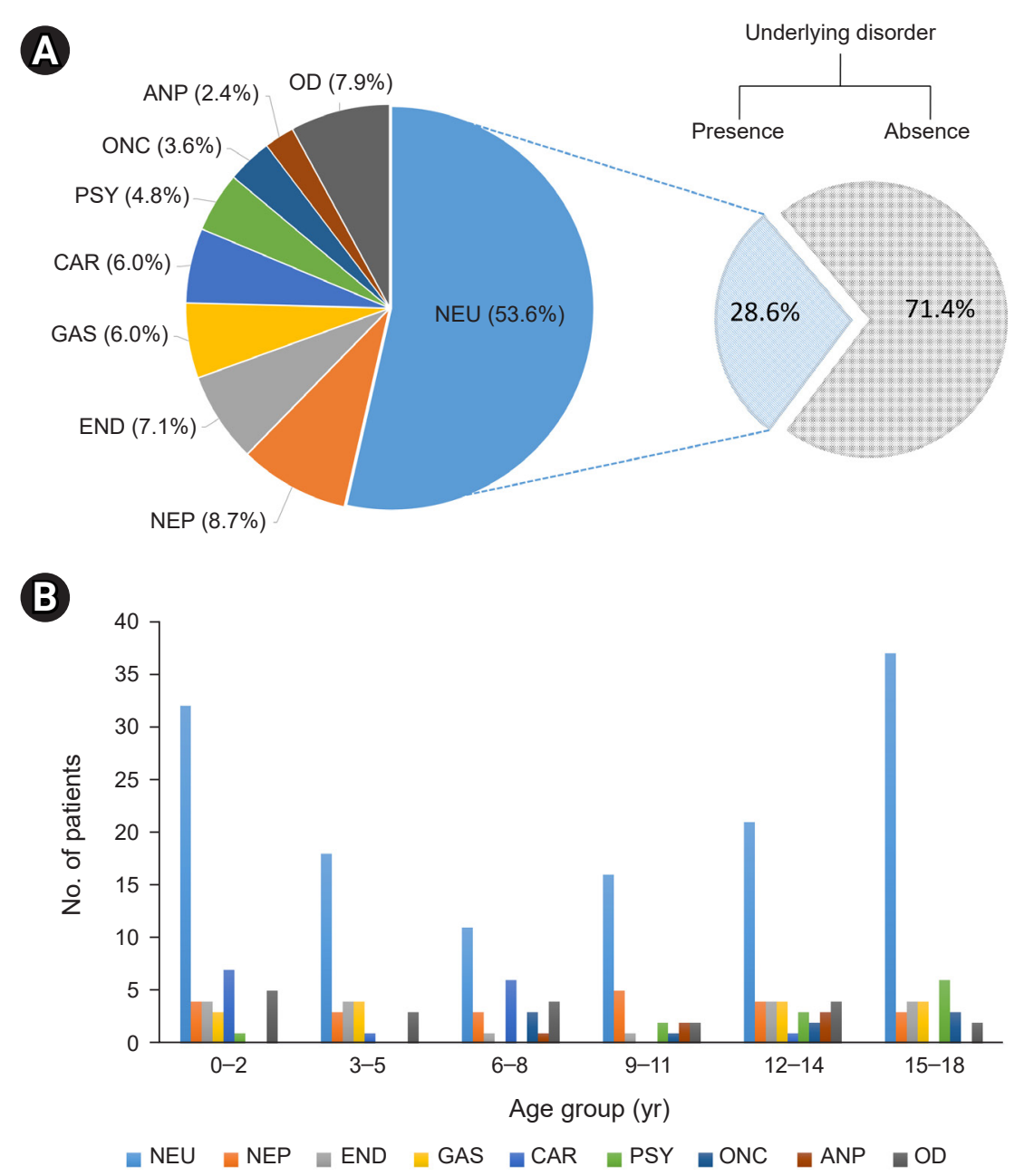

Figure 2. Underlying diseases of rhabdomyolysis. (A) Proportion of each underlying disorder. (B) Age distribution of the underlying disorder.

ANP, allergy and pulmonological disorders; CAR, cardiologic disorder; END, endocrinological and metabolic disorders; GAS, genetic and syndromic disorders; NEP, nephrological disorder; NEU, neurological disorder; ONC, oncologic disorder; OD, other disorders; PSY, psychological disorder; UD, underlying disorder.

\section{Laboratory data}

When the AKI and non-AKI subgroups were compared, most laboratory results showed significant differences between these two groups. A significantly greater proportion of AKI cases was associated with more positive urine occult blood (OB) $(p<0.001)$. The proportions of hematuria and urine myoglobin were higher in the AKI subgroup $(\mathrm{p}<0.001$ for both). Initial and peak CK and peak myoglobin levels were also significantly higher in the AKI subgroup, while the initial calcium levels were lower in the AKI subgroup. The initial and peak blood levels of parameters of interest, including aspartate aminotransferase (AST), alanine aminotransferase (ALT), lactate dehydrogenase (LDH), blood urea nitrogen (BUN), creatinine, uric acid, and phosphorus, were significantly higher in the AKI subgroup (Table 2).

Among the results of univariate analysis (Table 1,2), clinical information, which was initially assessable and whose p-value was less than 0.1 , underwent stepwise variable selection, and the following variables were selected: age subgroup, the presence of underlying disease, multiorgan failure, urine OB, CK, AST, uric acid, calcium, and phosphorus. Based on the multiple logistic regression model for predicting AKI in rhabdomyolysis, variables that were 


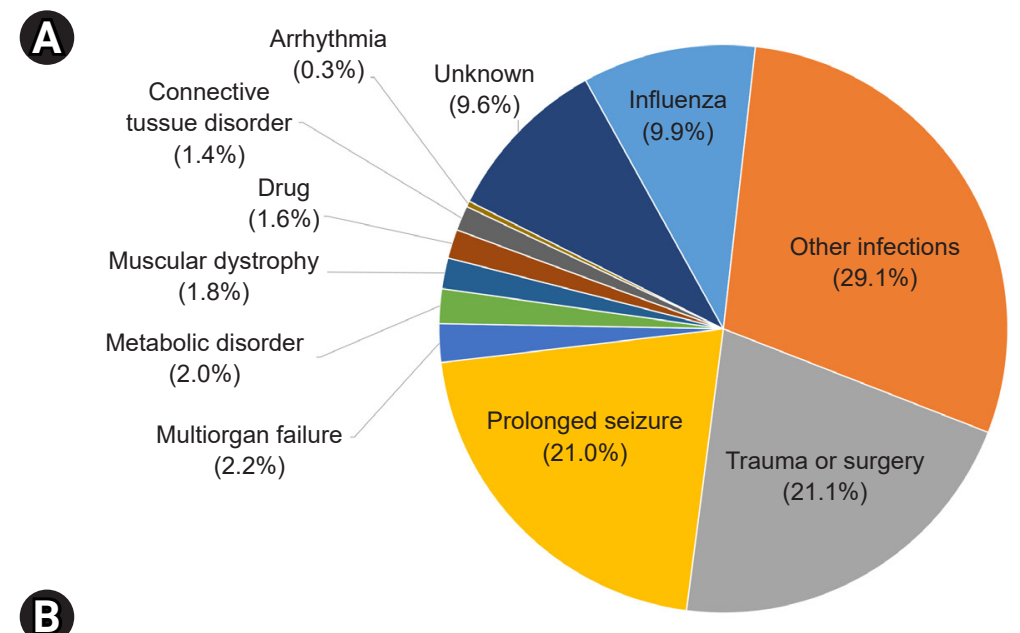

B

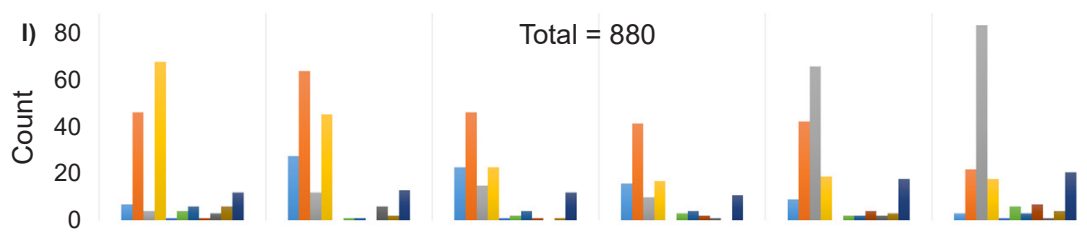

$\begin{array}{ll}\text { II) } 80 & \text { Non-AKI }=781\end{array}$
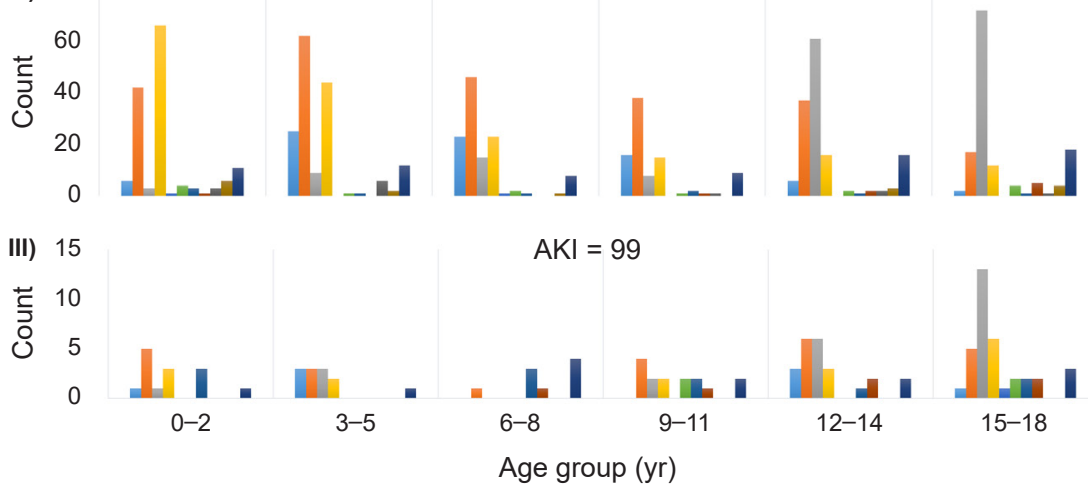

$\begin{array}{ll}\text { Influenza } & \text { Other infections } \\ \text { Trauma } & \text { Prolonged seizure } \\ \text { Arrhythmia } & \text { Metabolic disorder } \\ \square \text { Multiorgan failure } & \text { Drugs } \\ \square \text { Connective tissue disorder } & \text { Muscular dystrophy } \\ \square \text { Unknown } & \end{array}$

Figure 3. Cause of rhabdomyolysis. (A) Proportion of each cause. Infection, including both influenza and other infections, was the most prevalent cause (39.0\%). (B) Age distribution of the causes.

AKI, acute kidney injury.

independently associated with AKI development included the presence of underlying disease, multiorgan failure, urine OB, AST, uric acid, and calcium. Patients aged 15 to 18 years had an odds ratio of 2.3 relative to that of the reference group (3-5 years), but the p-value was insignificant. Urine OB 4+ and multiorgan failure-induced rhabdomyolysis had the highest odds ratios (Table 3). The discrimination value of the predictive model was established by means of an ROC curve, with an area under the ROC curve of 0.946 ( $p<$ 0.001) (Fig. 4).

\section{Outcomes}

CK and myoglobin were recovered within 5 to 6 days after 
Table 2. Laboratory results of acute rhabdomyolysis in those with and without AKI

\begin{tabular}{|c|c|c|c|c|}
\hline Variable & AKI $(n=99)$ & Non-AKI $(n=781)$ & Total $(n=880)$ & p-value \\
\hline \multicolumn{5}{|l|}{ Urine test } \\
\hline Urine occult blood & & & & $<0.001$ \\
\hline Negative & $27(27.3)$ & $544(69.7)$ & $571(64.9)$ & \\
\hline Trace & $0(0)$ & $12(1.5)$ & $12(1.4)$ & \\
\hline $1+$ & $9(9.1)$ & $54(6.9)$ & $63(7.2)$ & \\
\hline $2+$ & $17(17.2)$ & $52(6.7)$ & $69(7.8)$ & \\
\hline $3+$ & $38(38.4)$ & $106(13.6)$ & $144(16.4)$ & \\
\hline $4+$ & $8(8.1)$ & $13(1.7)$ & $21(2.4)$ & \\
\hline Urine RBC count (/HPF) & $34 \pm 67$ & $12 \pm 43$ & $15 \pm 46$ & 0.002 \\
\hline True hematuria, RBC $\geq 5$ /HPF & $45(45.5)$ & $105(13.4)$ & $150(17.0)$ & $<0.001$ \\
\hline Urine myoglobin, positive $(n=267)$ & $28(82.4)$ & $96(41.2)$ & $124(46.4)$ & $<0.001$ \\
\hline \multicolumn{5}{|l|}{ Blood test results at initial consult } \\
\hline $\mathrm{CK}(\mathrm{U} / \mathrm{L})$ & $3,161(1,050-12,772)$ & $1,986(1,078-5,698)$ & $2,144(1,077-6,262)$ & 0.05 \\
\hline Myoglobin (mg/mL) & $2,072(512-6,085)$ & $2,266(243-12,449)$ & $2,159(278-12,449)$ & 0.93 \\
\hline AST (U/L) & $179(78-973)$ & $86(49-213)$ & $90(50-263)$ & $<0.001$ \\
\hline $\operatorname{ALT}(U / L)$ & $88(24-382)$ & $34(17-120)$ & $36(17-138)$ & $<0.001$ \\
\hline $\mathrm{LDH}(\mathrm{U} / \mathrm{L})$ & $1,264(756-3,766)$ & $677(531-1,152)$ & 725 (542-1,354) & $<0.001$ \\
\hline BUN (mg/dL) & $25.4(15.5-53.5)$ & $10.8(8.3-13.5)$ & $11.2(8.4-14.7)$ & $<0.001$ \\
\hline Creatinine $(\mathrm{mg} / \mathrm{dL})$ & $1.5(0.9-3.0)$ & $0.5(0.4-0.7)$ & $0.5(0.4-0.8)$ & $<0.001$ \\
\hline Uric acid (mg/dL) & $8.0(5.6-12.5)$ & $4.2(3.2-5.9)$ & $4.5(3.2-6.3)$ & $<0.001$ \\
\hline Calcium (mg/dL) & $8.6(7.5-9.2)$ & $9.2(8.8-9.6)$ & $9.1(8.7-9.6)$ & $<0.001$ \\
\hline Phosphorus (mg/dL) & $5.1(4.1-6.5)$ & $4.4(3.9-5.1)$ & $4.5(3.9-5.2)$ & $<0.001$ \\
\hline \multicolumn{5}{|c|}{ Peak values recorded during the treatment period } \\
\hline CK $(U / L)$ & $6,562(2,340-21,127)$ & $2,685(1,425-7,777)$ & $2,859(1,452-9,090)$ & $<0.001$ \\
\hline Myoglobin $(\mathrm{mg} / \mathrm{mL})$ & $2,724(694-5,406)$ & $1,000(193-4,014)$ & $1,063(217-4,030)$ & 0.001 \\
\hline AST (U/L) & $406(110-1,933)$ & $112(65-318)$ & $120(67-397)$ & $<0.001$ \\
\hline $\operatorname{ALT}(U / L)$ & $244(50-809)$ & $49(26-176)$ & $53.50(27-221)$ & $<0.001$ \\
\hline $\mathrm{LDH}(\mathrm{U} / \mathrm{L})$ & $1,982(872-4,949)$ & $790(581-1,516)$ & $841(591-1,842)$ & $<0.001$ \\
\hline BUN (mg/dL) & $41.7(21.0-65.5)$ & $12.1(9.4-15.2)$ & $12.7(9.9-17.0)$ & $<0.001$ \\
\hline Creatinine $(\mathrm{mg} / \mathrm{dL})$ & $2.2(1.1-3.7)$ & $0.5(0.4-0.7)$ & $0.6(0.4-0.8)$ & $<0.001$ \\
\hline Uric acid (mg/dL) & $10.9(6.6-13.7)$ & $4.8(3.6-6.1)$ & $5.1(3.8-6.6)$ & $<0.001$ \\
\hline Calcium (mg/dL) & $9.4(9.1-10.1)$ & $9.6(9.1-10)$ & $9.5(9.1-10.0)$ & 0.57 \\
\hline Phosphorus (mg/dL) & $5.9(5.1-7.3)$ & $5(4.5-5.6)$ & $5.1(4.5-5.7)$ & $<0.001$ \\
\hline
\end{tabular}

Data are expressed as number (\%), mean \pm standard deviation, or median (interquartile range).

AKI, acute kidney injury; ALT, alanine aminotransferase; AST, aspartate aminotransferase; BUN, blood urea nitrogen; CK, creatine kinase; HPF, high-power field; $\mathrm{LDH}$, lactate dehydrogenase; RBC, red blood cell.

the first day of rhabdomyolysis, and there was no difference between the AKI and non-AKI subgroups. Short-term outcomes in the AKI subgroup were characterized by a higher proportion of volume overload ( $33.8 \%$ vs. $0.7 \%$; $p<0.001$ ) and longer hospitalization (18 days vs. 6 days; $\mathrm{p}<0.001$ ). The median oliguria recovery time was 2.5 days in the AKI subgroup. The long-term outcomes of 522 patients, excluding those with missing data, revealed a greater proportion of chronic kidney disease and recurrent rhabdomyolysis in the AKI subgroup $(\mathrm{p}<0.001)$ (Table 4).

\section{Complications}

Only $15.4 \%$ of patients with rhabdomyolysis experienced complications. The total incidence of AKI was 11.3\%; the incidence rates for the 0 to 9 years, 9 to 11 years, 12 to 14 years, and 15 to 18 years age subgroups were $6.4 \%$ to $7.9 \%, 11.3 \%$, $13.4 \%$, and $20.2 \%$, respectively. The AKI subgroup present- 
Table 3. Multiple logistic regression model for predicting AKI in patients with acute rhabdomyolysis

\begin{tabular}{|c|c|c|}
\hline Variable & OR $(95 \% \mathrm{Cl})$ & p-value \\
\hline Age group (yr) & Reference $=3-5 \mathrm{yr}^{\mathrm{c}}$ & \\
\hline $0-2$ & $0.96(0.32-2.87)$ & 0.95 \\
\hline $6-8$ & $0.84(0.24-2.89)$ & 0.78 \\
\hline $9-12$ & $0.72(0.21-2.44)$ & 0.59 \\
\hline $12-14$ & $1.15(0.43-3.1)$ & 0.79 \\
\hline $15-18$ & $2.30(0.89-5.93)$ & 0.09 \\
\hline Presence of underlying disorder & 3.35 (1.91-5.88) & $<0.001$ \\
\hline Multiorgan failure & $6.28(1.92-20.57)$ & 0.002 \\
\hline Urine occult blood $^{\mathrm{a}}$ & Reference $=$ negative & \\
\hline Trace & 0 (0-infinity) & $>0.99$ \\
\hline $1+$ & $2.05(0.78-5.38)$ & 0.15 \\
\hline $2+$ & $2.69(1.07-6.74)$ & 0.03 \\
\hline $3+$ & $4.46(2.22-8.97)$ & $<0.001$ \\
\hline $4+$ & $5.21(1.53-17.81)$ & 0.008 \\
\hline $\mathrm{CK} / 100^{\mathrm{a}, \mathrm{b}}$ & $1(1.00-1.00)$ & 0.43 \\
\hline AST $/ 100^{a, b}$ & $1.05(1.01-1.09)$ & 0.01 \\
\hline Uric acid ${ }^{a}$ & $1.23(1.14-1.34)$ & $<0.001$ \\
\hline Calcium $^{a}$ & $0.78(0.65-0.94)$ & 0.008 \\
\hline Phosphorus $^{\text {a }}$ & $1.03(0.89-1.19)$ & 0.73 \\
\hline
\end{tabular}

Initial creatinine was included in the multivariable logistic regression analysis to reveal uric acid, phosphorous, and calcium as risk factors for AKI, independent of creatinine.

AKI, acute kidney injury; AST, aspartate aminotransferase; Cl, confidence interval; CK, creatine kinase; OR, odds ratio.

a Laboratory findings at initial consult. ${ }^{\mathrm{D}} \mathrm{OR}$ of $\mathrm{CK} / 100$ and AST/100 can be interpreted as 100-unit increases in the values of CK and AST. 'Age range of 3 to 5 years was selected as the reference because this age group had the lowest incidence of AKI.

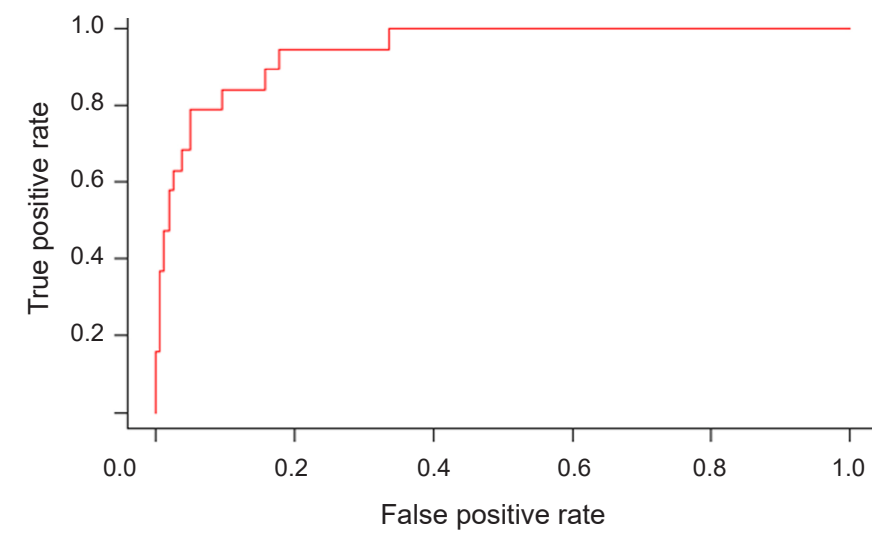

Figure 4. Receiver-operating characteristic curve of the multiple logistic regression model for predicting acute kidney injury.

ed a higher proportion of complications, such as mortality rate $(14.1 \%$ vs. $1.8 \%)$, disseminated intravascular coagulation ( $12.1 \%$ vs. $0.4 \%)$, and electrolyte disturbance $(25.3 \%$ vs. $3.7 \%$ ) (Table 4, Fig. 5).

\section{Treatment}

In this study, $24.2 \%$ of patients did not require treatment, with a significantly higher proportion of these patients existing in the non-AKI group ( $\mathrm{p}=0.002)$. Volume replacement was the most commonly used treatment $(72.1 \%)$. Alkalization and mannitol administration were performed significantly more often in the AKI subgroup $(p<0.001)$. Dialysis was performed in $31.3 \%$ of the 99 patients with AKI. Peritoneal dialysis was performed in only one patient, while the others required continuous renal replacement therapy (CRRT) (Table 5). The average dialysis period was 10.6 days.

\section{Discussion}

Rhabdomyolysis has been defined in various ways in previous studies. A CK level of at least $1,000 \mathrm{U} / \mathrm{L}$ or exceeding 5 times the upper limit of the normal range has been commonly used as the cutoff value for diagnosis [4], but those of at least 5,000 U/L and at least 10,000 U/L have also been 
Table 4. Outcomes and complications of acute rhabdomyolysis in patients with or without AKI

\begin{tabular}{|c|c|c|c|c|}
\hline Variable & $\mathrm{AKI}(\mathrm{n}=99)$ & Non-AKI $(n=781)$ & Total $(n=880)$ & $p$-value \\
\hline \multicolumn{5}{|l|}{ Short-term outcomes } \\
\hline CK recovery time (day) & $7.0(3.5-12.5)$ & $6.0(4.0-8.0)$ & $6.0(4.0-9.0)$ & 0.11 \\
\hline Myoglobin recovery time (day) & $5.0(3.0-11.0)$ & $5.0(3.0-9.0)$ & $5.0(3.0-9.0)$ & 0.44 \\
\hline Oliguria recovery time (day) & $2.5(2.0-8.0)$ & NA & $2.5(2.0-8.0)$ & 0.005 \\
\hline Volume overload, yes $(n=529)^{a}$ & $24(33.8)$ & $3(0.7)$ & $27(5.1)$ & $<0.001$ \\
\hline Duration of hospitalization (day) & $18.0(7.0-38.0)$ & $6.0(4.0-10.0)$ & $7.0(4.0-12.0)$ & $<0.001$ \\
\hline Chronic kidney disease & $3(6.4)$ & $0(0)$ & $3(0.6)$ & \\
\hline Recurrent rhabdomyolysis & $5(10.5)$ & $15(3.2)$ & $20(3.8)$ & \\
\hline \multicolumn{5}{|l|}{ Complications $^{\mathrm{b}}$} \\
\hline None & $10(10.1)$ & $734(94.0)$ & $744(84.6)$ & $<0.001$ \\
\hline Death & $14(14.1)$ & $14(1.8)$ & $28(3.2)$ & $<0.001$ \\
\hline
\end{tabular}

Data are expressed as median (interquartile range) or number (\%).

AKI, acute kidney injury; CK, creatine kinase; DIC, disseminated intravascular coagulation; NA, not applicable.

${ }^{\text {aT }}$ The number of patients except missing value. ${ }^{b}$ Multiple responses allowed the collection of data on complications.

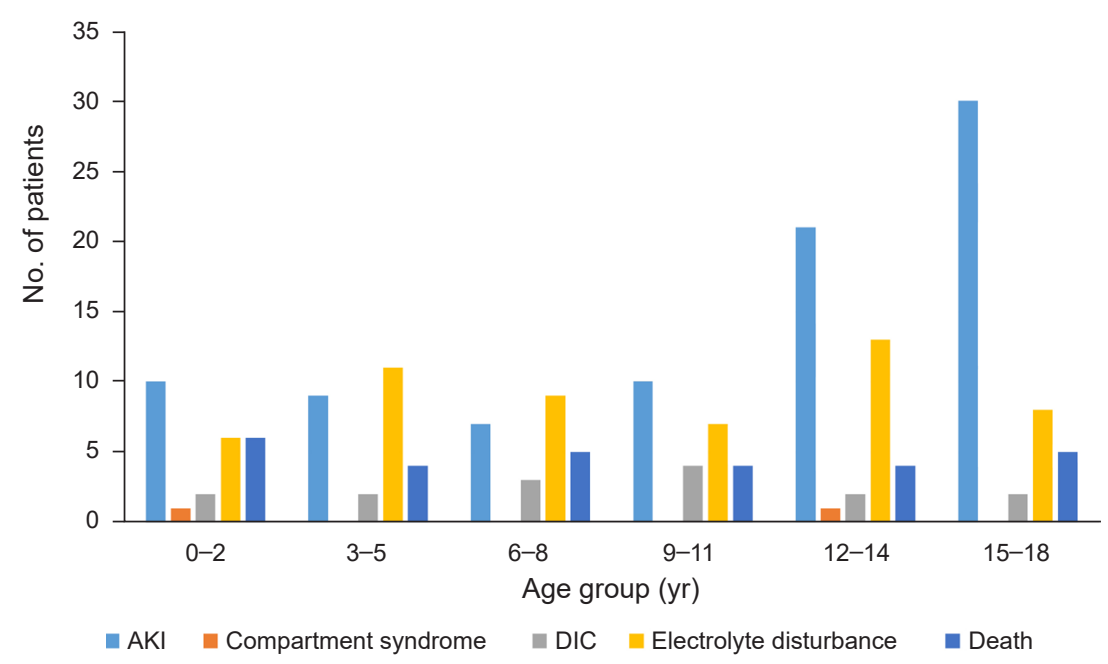

Figure 5. Distribution of complications by age in rhabdomyolysis patients.

AKI, acute kidney injury; DIC, disseminated intravascular coagulopathy.

adopted [8-10]. Another criterion that is less frequently used is myoglobin, whose reference level in previous studies ranged from at least $80 \mathrm{ng} / \mathrm{mL}$ to at least $150 \mathrm{ng} /$ $\mathrm{mL}[7,11,12]$. We used either CK or myoglobin level as the inclusion criteria for our study to maximize the number of participants.

Because of the diversity of definitions and subjects, the incidence of rhabdomyolysis varies from $4 \%$ to $50 \%$ in adult studies $[3,11]$. The exact incidence of pediatric rhabdomyolysis is unclear, but it has been reported to range from $5 \%$ to $37.5 \%$ [5-7]. Approximately $4 \%$ to $50 \%$ of cases of rhabdomyolysis lead to AKI, while up to $15 \%$ of AKI cases can be attributed to rhabdomyolysis [1-3]. The incidence of AKI in our study was $11.3 \%$. We believe that the use of a single, 
Table 5. Differences in treatment for patients with acute rhabdomyolysis with and without AKI

\begin{tabular}{lcccc}
\hline Treatment & AKI $(n=99)$ & Non-AKI $(n=781)$ & Total $(n=880)$ & $p$-value \\
\hline Volume replacement & $74(74.7)$ & $560(71.7)$ & $634(72.0)$ & 0.61 \\
Alkalization & $29(29.3)$ & $73(9.3)$ & $102(11.6)$ & $<0.001$ \\
Mannitol & $10(10.1)$ & $24(3.1)$ & $34(3.9)$ & 0.003 \\
Dialysis & $31(31.3)$ & $0(0)$ & $31(3.5)$ & $<0.001$ \\
No specific therapy & $11(11.1)$ & $202(25.9)$ & $213(24.2)$ & 0.002 \\
\hline
\end{tabular}

Data are expressed as number (\%).

Multiple responses allowed the collection of treatment method data.

AKI, acute kidney injury.

universal definition of rhabdomyolysis is necessary because it will aid in comparing incidence rates between studies and lead to advancements in this field.

A pediatric study performed in Korea investigated rhabdomyolysis in 39 children and reported the findings of a higher proportion of boys (2.5-fold more) among the affected children, epilepsy as the most common underlying disorder, and infection as the most common cause, similar to our results [7]. Another pediatric study that involved 172 children in Taiwan reported the existence of a higher proportion of boys (3.7-fold more) among the total cases and found viral myositis to be the most common cause. However, these authors also reported an absence of sex differences in AKI incidence [6]. Similarly, our study also revealed a male sex dominance. The higher proportion of adolescent males in rhabdomyolysis cases suggests that sex hormones or greater physical activity, which leads to more muscle mass, may contribute to the development of rhabdomyolysis. There were no sex differences in the proportion of causes, underlying disease, or complications of rhabdomyolysis in our study. Further studies are needed to determine whether sex contributes to rhabdomyolysis. We also did not observe any differences in the incidence of AKI according to sex. Therefore, male sex is not a risk factor for rhabdomyolysis-induced AKI.

The influence of anthropometric data on rhabdomyolysis and AKI has not been fully elucidated, and reports on the association between rhabdomyolysis and obesity are limited. Increased BMI could reflect a large muscle mass but usually implies excess adipose tissue that could induce pathological changes in the kidney and increase baseline circulating levels of nephrotoxic inflammatory molecules [13]. Several adult studies have indicated that obesity and high BMI are linked to rhabdomyolysis and AKI [14-17]. In a single-center cohort study on obesity and AKI unrelated to rhabdomyolysis, obese patients were more likely to develop AKI, and each $5-\mathrm{kg} / \mathrm{m}^{2}$ increase in BMI was associated with a 10\% greater risk of severe AKI [14]. However, BMI was not associated with AKI in a previous pediatric study [6], and the z-scores of height, weight, and BMI did not differ between the AKI and non-AKI subgroups in our study. At this time, the reason why there is a contradiction regarding the influence of obesity on rhabdomyolysis and AKI between adults and children remains unclear. With respect to the different results between pediatric and adult studies, limitations of BMI, body composition, and duration of obesity might affect the risk of AKI.

Although myalgia, weakness, and dark-colored urine have been known as the classic triad of symptoms of rhabdomyolysis, these findings are observed in less than $10 \%$ of affected patients. In a previous report, more than $50 \%$ of patients did not have myalgia or weakness, while dark-colored urine was observed in only $3.6 \%$ of the cases [2]. Our cases showed a relatively greater proportion of the classic triad than found in previous studies, but these symptoms were not distinguishing features of the AKI subgroup.

It is well known that infants and toddlers are vulnerable to febrile illness and seizures and that outside activities and accidents tend to increase in adolescents. The etiologic distribution of bimodal distribution in our study reflects these characteristics and may influence the frequency of rhabdomyolysis in each age group. Therefore, these etiological results vary depending upon the subpopulation. Previous adult studies suggested that trauma and illicit drug abuse are primary causes of rhabdomyolysis [18-20]. In our study, trauma was the second most common cause of rhabdomyolysis and was more prevalent in adolescent patients. However, drug-induced rhabdomyolysis accounted for only $1.7 \%$ of cases, and there were no cases of illicit drug use. Relatively low frequencies of trauma-induced 
and drug-induced rhabdomyolysis are believed to reflect the characteristics of the pediatric population and low prevalence of drug abuse in Korea [21,22]. Therefore, we suggest that the causes of rhabdomyolysis should be investigated in specific subpopulations.

Patients who experience seizures are considered vulnerable to rhabdomyolysis for two reasons. First, seizures lead to postictal alterations in several blood parameters, such as CK [23]. It is well known that skeletal muscle injury can be caused by convulsive seizures or status epilepticus [24]. Second, the majority of antiepileptic drugs, such as valproic acid and levetiracetam, are relevant to rhabdomyolysis [25]. Some neuromuscular disorders, such as muscular dystrophy, are accompanied by increased CK levels, which are susceptible to triggering factors such as fever, exercise, bisphosphonate use, and anesthesia [26-28]. A retrospective study reported 13 patients with rhabdomyolysis featuring muscular dystrophy; the median duration between the first episodes of rhabdomyolysis and genetic diagnosis was 2 years, and the authors suggested that persistently increased CK levels with recurrent rhabdomyolysis warrant a workup for underlying muscular dystrophy [28].

Muscle-derived components are effective biomarkers for renal damage, resulting in hyperphosphatemia; hypocalcemia; hyperuricemia; increased plasma LDH and AST levels; and increased urinary excretion of creatinine, uric acid, and glucose [29]. Although CK is a cornerstone of rhabdomyolysis diagnosis, it remains controversial whether CK itself is related to the risk of AKI and mortality. Some studies have shown that the initial or peak CK value is not a reliable marker of AKI or mortality outcome [30-32]. However, other studies have suggested that the initial and peak values of CK and myoglobin are risk factors for AKI [6,20]. Our study showed that the initial CK level was significantly increased, but that of myoglobin was not. These contradictory results regarding CK and myoglobin may be affected by differences in the time interval from symptom onset to arrival at the hospital. CK levels rise in rhabdomyolysis within 12 hours of the onset of muscle injury, peak within 24 to 72 hours, and normalize approximately 5 days after the cessation of muscle injury. The half-life of CK is approximately 36 hours [33]. Meanwhile, myoglobin has a short half-life (2-4 hours) and may return to normal within 6 to 8 hours [2]. In future studies, it is worth considering adjusting the initial CK level according to the timing of muscle injury.
Our study revealed significant differences in most traditional biomarkers, including AST, ALT, LDH, uric acid, calcium, and phosphorus. Although they were not included in our study, constant albuminuria and hypoalbuminemia have been suggested as independent risk factors for AKI in previous research $[7,20]$. In this respect, timely measurements of traditional biomarkers are useful and practical for predicting AKI. The McMahon score predicts the risk of renal failure requiring renal replacement therapy (RRT) or resulting in mortality in patients with rhabdomyolysis. The variables include age; female sex; origin of rhabdomyolysis; and initial values of creatinine, calcium, CK, and phosphate. A McMahon score of 6 points or higher has greater sensitivity and specificity than a CK level of greater than 5,000 U/L in predicting the risk of RRT [34]. Although the McMahon score was developed for adult patients, modification of variables based on our and other pediatric studies may give us a scoring system applicable to children in future studies.

Dipstick urine OB can yield false-positive results caused by dehydration, exercise, hemoglobinuria, and myoglobinuria, which are commonly seen in rhabdomyolysis $[1,35]$. However, it is an inexpensive and rapid test that is useful for detecting the risk-estimation markers of AKI. The correlation between AKI and urine OB shown in our study supports the usefulness of the urine dipstick test. In our study, dark-colored urine did not show any difference between the AKI-status subgroups, while true hematuria was significantly higher in the AKI subgroup. Thus, the influence of hematuria on the degree of urine $\mathrm{OB}$ is greater than that of myoglobin in this group, and these results suggest a role of hematuria in AKI. Hematuria has a pathophysiological mechanism that is involved in aspects of renal damage, such as direct tubular damage, oxidative stress, and secretion of inflammatory cytokines, which occurs during rhabdomyolysis [36]. Hematuria is presumed to aggravate kidney function in patients with rhabdomyolysis. Therefore, the risk of AKI could increase in cases of true hematuria and patients with higher OB scores following urinalysis.

Fluid resuscitation has been widely used to treat rhabdomyolysis, and it was also the most commonly used method in our study. Although fluid therapy is emphasized by consensus $[5,37,38]$, other treatment methods are still controversial. Dawley [37] reported that management consists of rapidly aggressive intravenous resuscitation to maintain urine output and limited use of bicarbonate for acidosis 
and mannitol for oliguria, respectively. Michelsen et al. [38] reported guidelines that recommend early fluid resuscitation using crystalloids but not the routine use of diuretics, mannitol, alkalization, or RRT. CRRT effectively removes myoglobin and manages AKI [38]. A Cochrane systematic review concluded that CRRT provides some benefits, but the evidence is insufficient [39]. However, significantly decreased myoglobin levels; improved BUN, creatinine, and potassium levels; and reduced oliguria and hospitalizations were reported relative to when conventional therapy was used. Mortality rates exhibited significant differences, but data on long-term outcomes are lacking [39].

To our knowledge, this is the first large-scale pediatric multicenter study. First, our study clarified the age distribution, proportions of boys and girls, and anthropometric data in rhabdomyolysis, which have been reported in previous studies. Second, our detailing of underlying diseases and causes of rhabdomyolysis can help in understanding their influence in the pediatric population. Third, we found that the independent risk factors for AKI in rhabdomyolysis were multiorgan failure; the presence of underlying disorders; increased levels of urine $\mathrm{OB}$, AST, and uric acid; and decreased levels of calcium. Unlike in previous adult studies, sex, BMI, and CK were not included as risk factors. Fourth, the role of urinalysis was newly highlighted for predicting AKI.

However, there are some limitations to account for as this was a retrospective multicenter study. First, some medical record data regarding etiology, underlying disorders, and laboratory data were missing, which led to the exclusion of some cases. Second, AKI and oliguria could be underestimated or overestimated because of the methodological issues with baseline creatinine level and urine output. Third, some laboratory data, such as CK and myoglobin levels and urine red blood cell count exceeding the upper reference limit, were reported only as values over the upper limit instead of the exact value, which might have led to underestimation of their influence. In addition, the analysis methods and equipment used likely varied among the involved hospitals. Fourth, underlying disorders were classified based on the involved organ or pathology of the disease for the purpose of detailed descriptions, but the criteria for classification were vague for disorders with multisystemic involvement, and severity was not considered. Thus, the interpretation of the influence of underlying disorders on rhabdomyolysis and AKI is limited.

Rhabdomyolysis is a well-known disease, but its epidemiology, risk factors, incidence rates of AKI, and mortality have not been elucidated because of the heterogeneity caused by varying definitions and etiologies. Our study revealed characteristic clinical and laboratory features of rhabdomyolysis in a multicenter Korean pediatric population as well as the predictive factors for AKI. These findings will contribute to a broader understanding of pediatric rhabdomyolysis and enable early intervention against rhabdomyolysis-induced AKI.

\section{Additional information}

${ }^{1}$ Department of Pediatrics, Pusan National University School of Medicine, Busan, Republic of Korea

${ }^{2}$ Department of Pediatrics, Kyungpook National University, School of Medicine, Daegu, Republic of Korea

${ }^{3}$ Department of Pediatrics, National Police Hospital, Seoul, Republic of Korea

${ }^{4}$ Department of Pediatrics, Chonnam National University Hospital, Gwangju, Republic of Korea

${ }^{5}$ Department of Pediatrics, Korea University College of Medicine, Seoul, Republic of Korea

${ }^{6}$ Department of Pediatrics, Inha University School of Medicine, Incheon, Republic of Korea

${ }^{7}$ Department of Pediatrics, Yonsei University College of Medicine, Seoul, Republic of Korea

${ }^{8}$ Department of Pediatrics, Chungbuk National University College of Medicine, Cheongju, Republic of Korea

${ }^{9}$ Department of Pediatrics, Yeungnam University College of Medicine, Daegu, Republic of Korea

${ }^{10}$ Department of Pediatrics, Ewha Womans University College of Medicine, Seoul, Republic of Korea

${ }^{11}$ Department of Pediatrics, National Health Insurance Corporation Ilsan Hospital, Goyang, Republic of Korea

${ }^{12}$ Department of Pediatrics, Samsung Medical Center, Seoul, Republic of Korea

${ }^{13}$ Department of Pediatrics, Dankook University College of Medicine, Cheonan, Republic of Korea

${ }^{14}$ Department of Pediatrics, The Catholic University of Korea, Bucheon Saint Mary's Hospital, Bucheon, Republic of Korea

${ }^{15}$ Department of Pediatrics, Jeju National University School of Medicine, Jeju, Republic of Korea

${ }^{16}$ Department of Pediatrics, The Catholic University of Korea, Saint Vincent's Hospital, Suwon, Republic of Korea

${ }^{17}$ Department of Pediatrics, Seoul National University College of Medicine, Seoul, Republic of Korea

${ }^{18}$ Department of Pediatrics, Hallym University Sacred Heart Hospital, Anyang, Republic of Korea

${ }^{19}$ Department of Pediatrics, Yonsei University Wonju College of Medicine, Wonju, Republic of Korea

${ }^{20}$ Department of Pediatrics, Gachon University Gil Medical Center, Incheon, Republic of Korea

${ }^{21}$ Department of Pediatrics, Ajou University School of Medicine, 
Suwon, Republic of Korea

${ }^{22}$ Department of Pediatrics, Samsung Changwon Hospital, Changwon, Republic of Korea

${ }^{23}$ Department of Pediatrics, Konkuk University School of Medicine, Seoul, Republic of Korea

${ }^{24}$ Department of Pediatrics, Children's Hospital, Asan Medical Center, Seoul, Republic of Korea

\section{Conflicts of interest}

All authors have no conflicts of interest to declare.

\section{Authors' contributions}

Conceptualization: HSB, EMY, KHY, SH, JIS

Data curation, Formal analysis: SY, KHL, HSL, KMJ, KHK, MJL, KHH, HSH, ISH

Investigation: All authors

Methodology: JWL, SJK, TSH, HIC, HGK, MKN, HKC

Writing-original draft: SY, JYS, MHC, SHK, HC, JSS, JHO, STL, KSK, JHL, YSP

Writing-review \& editing: SHK, MHC, KML

All authors read and approved the final manuscript.

\section{ORCID}

Sukdong Yoo, https://orcid.org/0000-0003-2365-9134 Min Hyun Cho, https://orcid.org/0000-0002-7965-7587 Hee Sun Baek, https://orcid.org/0000-0003-0940-360X Ji Yeon Song, https://orcid.org/0000-0002-9665-4177 Hye Sun Lee, https://orcid.org/0000-0002-2790-2448 Eun Mi Yang, https://orcid.org/0000-0001-9410-5855 Kee Hwan Yoo, https://orcid.org/0000-0001-6490-4293 Su Jin Kim, https://orcid.org/0000-0003-0893-0512 Jae Il Shin, https://orcid.org/0000-0003-2326-1820 Keum Hwa Lee, https://orcid.org/0000-0002-1511-9587 Tae-Sun Ha, https://orcid.org/0000-0002-1249-9342 Kyung Mi Jang, https://orcid.org/0000-0002-2226-9268 Jung Won Lee, https://orcid.org/0000-0003-1846-3153 Kee Hyuck Kim, https://orcid.org/0000-0003-4720-6455 Heeyeon Cho, https://orcid.org/0000-0003-3137-6054 Mee Jeong Lee, https://orcid.org/0000-0002-7504-1232 Jin-Soon Suh, https://orcid.org/0000-0002-6566-6618 Kyoung Hee Han, https://orcid.org/0000-0002-6830-7311 Hye Sun Hyun, https://orcid.org/0000-0001-8525-1471 Il-Soo Ha, https://orcid.org/0000-0001-5428-6209
Hae Il Cheong, https://orcid.org/0000-0001-7556-1265 Hee Gyung Kang, https://orcid.org/0000-0001-8323-5320 Mee Kyung Namgoong, https://orcid.org/0000-0002-8156-8970 Hye-Kyung Cho, https://orcid.org/0000-0003-0990-1350 Jae-Hyuk Oh, https://orcid.org/0000-0002-8827-7703 Sang Taek Lee, https://orcid.org/0000-0003-2570-3290 Kyo Sun Kim, https://orcid.org/0000-0001-9322-6508 Joo Hoon Lee, https://orcid.org/0000-0001-8010-3605 Young Seo Park, https://orcid.org/0000-0001-7653-2036 Seong Heon Kim, https://orcid.org/0000-0001-8003-3010

\section{References}

1. Panizo N, Rubio-Navarro A, Amaro-Villalobos JM, Egido J, Moreno JA. Molecular mechanisms and novel therapeutic approaches to rhabdomyolysis-induced acute kidney injury. Kidney Blood Press Res 2015;40:520-532.

2. Torres PA, Helmstetter JA, Kaye AM, Kaye AD. Rhabdomyolysis: pathogenesis, diagnosis, and treatment. Ochsner J 2015;15:5869.

3. Bosch X, Poch E, Grau JM. Rhabdomyolysis and acute kidney injury. N Engl J Med 2009;361:62-72.

4. Chavez LO, Leon M, Einav S, Varon J. Beyond muscle destruction: a systematic review of rhabdomyolysis for clinical practice. Crit Care 2016;20:135.

5. Szugye HS. Pediatric Rhabdomyolysis. Pediatr Rev 2020;41:265275.

6. Wu CT, Huang JL, Lin JJ, Hsia SH. Factors associated with nontraumatic rhabdomyolysis and acute renal failure of children in Taiwan population. Pediatr Emerg Care 2009;25:657-660.

7. Lim YS, Cho H, Lee ST, Lee Y. Acute kidney injury in pediatric patients with rhabdomyolysis. Korean J Pediatr 2018;61:95-100.

8. Kim JH, Yun S, Hwang SS, et al. The 2017 Korean National Growth Charts for children and adolescents: development, improvement, and prospects. Korean J Pediatr 2018;61:135-149.

9. Gaião S, Cruz DN. Baseline creatinine to define acute kidney injury: is there any consensus? Nephrol Dial Transplant 2010; 25:3812-3814.

10. Kellum JA, Lameire N, Aspelin P, et al. Kidney disease: improving global outcomes (KDIGO) acute kidney injury work group. KDIGO clinical practice guideline for acute kidney injury. Kidney Int Suppl 2012;2:1-138.

11. Bagley WH, Yang H, Shah KH. Rhabdomyolysis. Intern Emerg Med 2007;2:210-218.

12. Kuzmanovska B, Cvetkovska E, Kuzmanovski I, et al. Rhabdomy- 
olysis in critically ill surgical patients. Med Arch 2016;70:308-310.

13. Kovesdy CP, Furth S, Zoccali C; World Kidney Day Steering Committee. Obesity and kidney disease: hidden consequences of the epidemic. Physiol Int 2017;104:1-14.

14. Danziger J, Chen KP, Lee J, et al. Obesity, acute kidney injury, and mortality in critical illness. Crit Care Med 2016;44:328-334.

15. Chakravartty S, Sarma DR, Patel AG. Rhabdomyolysis in bariatric surgery: a systematic review. Obes Surg 2013;23:1333-1340.

16. Grigorian A, Gabriel V, Nguyen NT, et al. Black race and body mass index are risk factors for rhabdomyolysis and acute kidney injury in trauma. J Invest Surg 2020;33:283-290.

17. Vasquez CR, DiSanto T, Reilly JP, et al. Relationship of body mass index, serum creatine kinase, and acute kidney injury after severe trauma. J Trauma Acute Care Surg 2020;89:179-185.

18. Linares LA, Golomb BA, Jaojoco JA, Sikand H, Phillips PS. The modern spectrum of rhabdomyolysis: drug toxicity revealed by creatine kinase screening. Curr Drug Saf2009;4:181-187.

19. Herráez García J, Torracchi Carrasco AM, Antolí-Royo AC, de la Fuente Blanco R, Santos Jiménez MT. Rhabdomyolysis: a descriptive study of 449 patients. Med Clin (Barc) 2012;139:238242.

20. Rodríguez E, Soler MJ, Rap O, Barrios C, Orfila MA, Pascual J. Risk factors for acute kidney injury in severe rhabdomyolysis. PLoS One 2013;8:e82992.

21. Feng LY, Yu WJ, Chang WT, Han E, Chung H, Li JH. Comparison of illegal drug use pattern in Taiwan and Korea from 2006 to 2014. Subst Abuse Treat Prev Policy 2016;11:34.

22. Hong CH. Current health issues in Korean adolescents. Korean J Pediatr 2011;54:395-400.

23. Nass RD, Sassen R, Elger CE, Surges R. The role of postictal laboratory blood analyses in the diagnosis and prognosis of seizures. Seizure 2017;47:51-65.

24. Zhou J, Luo B, Peng G. A single seizure attack induced rhabdomyolysis. Neurol India 2017;65(Suppl):S93-S94.

25. Jiang W, Wang X, Zhou S. Rhabdomyolysis induced by antiepileptic drugs: characteristics, treatment and prognosis. Expert Opin Drug Saf 2016;15:357-365.

26. Lemon J, Turner L, Dharmaraj P, Spinty S. Rhabdomyolysis and myoglobinuria following bisphosphonate infusion in patients with Duchenne muscular dystrophy. Neuromuscul Disord 2019;
29:567-568.

27. Hayes J, Veyckemans F, Bissonnette B. Duchenne muscular dystrophy: an old anesthesia problem revisited. Paediatr Anaesth 2008;18:100-106.

28. Lahoria R, Milone M. Rhabdomyolysis featuring muscular dystrophies. J Neurol Sci 2016;361:29-33.

29. Gabow PA, Kaehny WD, Kelleher SP. The spectrum of rhabdomyolysis. Medicine (Baltimore) 1982;61:141-152.

30. Kamal F, Snook L, Saikumar JH. Rhabdomyolysis-associated acute kidney injury with normal creatine phosphokinase. Am J Med Sci 2018;355:84-87.

31. Baeza-Trinidad R, Brea-Hernando A, Morera-Rodriguez S, et al. Creatinine as predictor value of mortality and acute kidney injury in rhabdomyolysis. Intern Med J 2015;45:1173-1178.

32. Gelbart B, DeMarco R, David Hussey A, et al. Rhabdomyolysis in a tertiary PICU: a 10-year study. Pediatr Crit Care Med 2018;19:e51-e57.

33. Subramanian A, Sukheeja D, Trikha V, Pandey AK, Albert V, Pandey RM. Evaluation of serum creatine kinase and urinary myoglobin as markers in detecting development of acute renal failure in severely injured trauma patients. ISRN Emerg Med 2013;2013:241036.

34. Simpson JP, Taylor A, Sudhan N, Menon DK, Lavinio A. Rhabdomyolysis and acute kidney injury: creatine kinase as a prognostic marker and validation of the McMahon Score in a 10-year cohort: a retrospective observational evaluation. Eur J Anaesthesiol 2016;33:906-912.

35. Simerville JA, Maxted WC, Pahira JJ. Urinalysis: a comprehensive review. Am Fam Physician 2005;71:1153-1162.

36. Moreno JA, Sevillano Á, Gutiérrez E, et al. Glomerular hematuria: cause or consequence of renal inflammation? Int J Mol Sci 2019;20:2205.

37. Dawley C. Myalgias and myopathies: rhabdomyolysis. FP Essent 2016;440:28-36.

38. Michelsen J, Cordtz J, Liboriussen L, et al. Prevention of rhabdomyolysis-induced acute kidney injury: a DASAIM/DSIT clinical practice guideline. Acta Anaesthesiol Scand 2019;63:576-586.

39. Zeng X, Zhang L, Wu T, Fu P. Continuous renal replacement therapy (CRRT) for rhabdomyolysis. Cochrane Database Syst Rev 2014;(6):CD008566. 